\title{
Usefulness of the Modified Lavage Technique of Bingisser and KL-6 Monitoring in a Patient with Pulmonary Alveolar Proteinosis
}

\author{
Nobuhisa Ishikawa, Keiichi Kondo, Tetsuya Oguri, Masakazu Kamitsuna, Joji SaKurai, Kazunori Fujtaka, \\ Masahiro Yamasaki, Hiroyuki MaEDA*, Takeshi Isobe and Nobuoki KoHNo
}

\begin{abstract}
A 48-year-old man diagnosed as pulmonary alveolar proteinosis (PAP) received a whole-lung lavage by the conventional lavage technique, but he failed to show any clinical or functional improvement. Therefore, we applied a modified lavage technique of Bingisser resulting in the impressive clinical and functional improvement. We measured the levels of KL-6 in bronchoalveolar lavage fluid (BALF) at selected time intervals. KL-6 levels in BALF were higher during the lavage by the modified lavage technique of Bingisser than the conventional lavage technique. We were able to verify the usefulness of the modified lavage technique of Bingisser by monitoring the levels of KL-6 in BALF. The modified lavage technique of Bingisser may be useful for PAP treatment.
\end{abstract}

(Internal Medicine 41: 381-385, 2002)

Key words: krebs der Lungen-6, MUC1, epithelial lining fluid

\section{Introduction}

Pulmonary alveolar proteinosis (PAP) is characterized by the filling of alveoli with periodic acid-Schiff positive proteinaceous materials and by hypertrophy of type II pneumocytes. Alveolar accumulation of proteinaceous materials is thought to be due to overproduction by type II pneumocytes or to decreased surfactant clearance because of a dysfunction of alveolar macrophages $(1,2)$. To date, a whole-lung lavage has been the only effective treatment for PAP (3); however, some patients may not improve despite repeated and extensive therapeutic lavages $(4,5)$. Recently, Bingisser et al $(6)$ reported that manual ventilation applied during the second half of the drainage cycle might aid the removal of proteinaceous materials.
Krebs der Lungen-6 (KL-6) is a MUC1 mucin (molecular weight $>1,000 \mathrm{kDa}$ ) that has been classified as a cluster $9 \mathrm{ex}$ pressed on lung tumors and a differentially expressed tumor antigen (7-9). Recently, KL-6 levels in the serum and bronchoalveolar lavage fluid (BALF) have been useful for the diagnosis of patients with PAP and to estimate the extent of the disease $(10,11)$.

In this report, we confirmed the effectiveness of the modified lavage technique of Bingisser et al for a patient with PAP, and monitored serum and BALF KL-6 levels before and after the therapeutic lavages.

\section{Case Report}

In 1995, chest radiography on a routine medical examination of a 48-year-old man revealed possible interstitial pneumonia (IP). He was a non-smoker and had no environmental history of occupational exposure to dusts. Chest X-ray and computed tomography (CT) were performed at 6-month intervals following the initial examination. In January 1999, he suffered from exertional dyspnea, which was not resolved despite oral administration of prednisolone $20 \mathrm{mg}$ /day started from May 1999. He was admitted to Hiroshima University Hospital in November 1999.

Physical examination upon admission revealed that heart sounds were clear; however, mild fine crackles were audible in both lower lung fields. Arterial blood gas analysis under room air revealed a $\mathrm{PaO}_{2}$ of $61.2 \mathrm{mmHg}$ and a $\mathrm{PaCO}_{2}$ of $36.9 \mathrm{mmHg}$, with a pH of 7.434. Hematological examination showed normal leukocyte counts, with normal differentials, and a mild increase in hemoglobin levels $(17.4 \mathrm{~g} / \mathrm{dl})$. Laboratory examinations showed increased serum levels of lactate dehydrogenase (LDH) (717 IU/l; normal range, 240-530 IU/l), KL-6 $(5,860 \mathrm{U} / \mathrm{ml}$; normal range, $240-530 \mathrm{U} / \mathrm{ml})$, and carcinoembryonic antigen (CEA) $(21.8 \mathrm{ng} / \mathrm{ml}$; normal range, $0.6-4.0 \mathrm{ng} / \mathrm{ml})$. A chest $\mathrm{X}$-ray revealed diffuse bilateral reticular shadows pri-

From the Second Department of Internal Medicine, Hiroshima University Faculty of Medicine, Hiroshima and *the Department of Respiratory Division, Hiroshima Prefecture Hospital, Hiroshima

Received for publication; July 11, 2001; Accepted for publication December 20, 2001

Reprint requests should be addressed to Dr. Keiichi Kondo, the Second Department of Internal Medicine, Hiroshima University Faculty of Medicine, 1-2-3 Kasumi, Minami-ku, Hiroshima 734-8551 
marily involving the middle and lower lung fields. Chest CT revealed diffuse ground-glass opacity with a thickening of the interlobular wall and a clear peripheral zone (Fig. 1A). We performed bronchoalveolar lavage and transbronchial biopsy using a flexible bronchoscope and thereby diagnosed his disease to be PAP.

Following the diagnosis, we performed two segmental left lower-lobe lavages by a bronchoscope once a week, but no clinical or radiographic improvement was observed. In February 2000, we performed a conventional whole-lung lavage of the left lung using a double lumen endotracheal tube under general anesthesia, as previously described (1). He received a detailed explanation of the study protocol and gave written informed consent before each therapeutic lavage. After degassing the nondependent lung, we started single-lung ventilation of the dependent lung with a $\mathrm{FiO}_{2}$ of 1.0 and low positive endexpiratory pressure (PEEP) of $2 \mathrm{~cm} \mathrm{H}_{2} \mathrm{O}$. Lavage was performed by gravitational infusion $\left(30 \mathrm{~cm} \mathrm{H}_{2} \mathrm{O}\right)$ and drainage of warm saline at $37^{\circ} \mathrm{C}$ in sequential aliquots of $1.0 \mathrm{l}$. To enhance lavage recovery, chest percussion and positional drainage were performed during the therapeutic lavage. These procedures were repeated until the effluent became clear. Two weeks later, how- ever, we did not observe any clinical or functional improvement (Fig. 1B). We then performed 12 lavages by the conventional lavage technique followed by 4 lavages by the modified lavage technique of Bingisser et al, which is described below (Fig. 2). First, $1.0 \mathrm{l}$ of warm saline was instilled into the lavaged lung. After passive recovery of half the amount of fluid, we manually ventilated the dependent lung 20 times with a tidal volume of $500 \mathrm{ml}$. The rest of the fluid was then recovered and the procedure was performed a total of 4 times. In April 2000, we performed a right whole-lung lavage, which consisted of 8 lavages by the conventional lavage technique followed by 3 lavages by the modified lavage technique of Bingisser et al (6). This modified technique clearly led to clinical and functional improvement (Fig. 1C).

We monitored KL-6 levels in serum and BALF by a sandwich-type enzyme-linked immunosorbent assay (ELISA) (7). Blood samples were collected at the following time points: at 30 minutes intervals during each therapeutic lavage and at 0,3 , and 6 hours after completing the whole-lung lavage. BALF samples were collected from all BALF returns during each therapeutic lavage. Initial returns of BALF appeared milky but gradually became clear after repeated therapeutic lavages. The
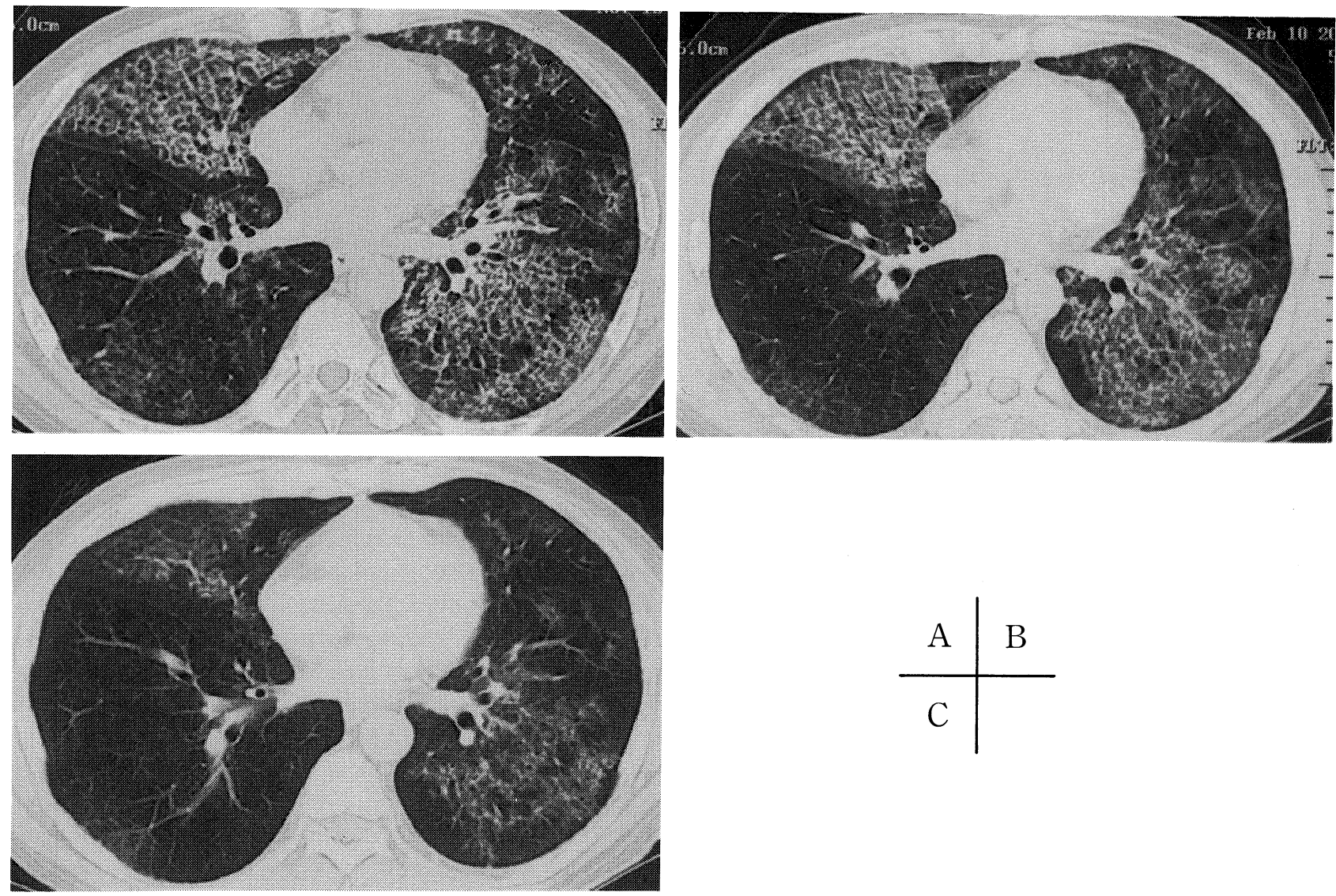

Figure 1. (A) Chest CT scan before 1st whole-lung lavage revealed diffuse ground glass opacity with a thickening of the interlobular wall. (B) Chest CT after 1st whole-lung lavage revealed a slight reduction of ground glass oppacity. (C) Chest CT after 3rd whole-lung lavage, revealed a significant reduction of ground-glass oppacity. 


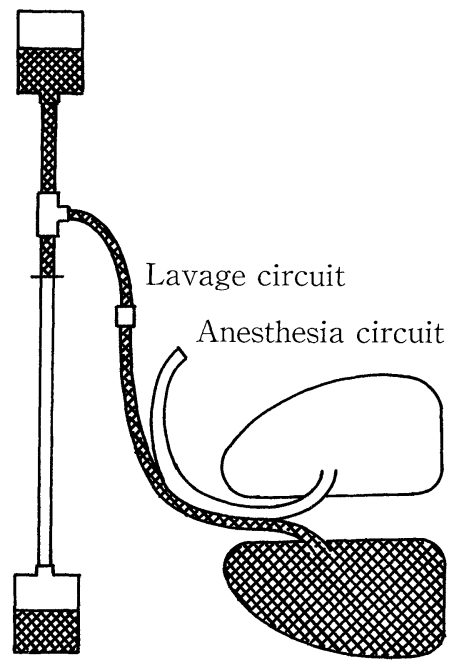

A

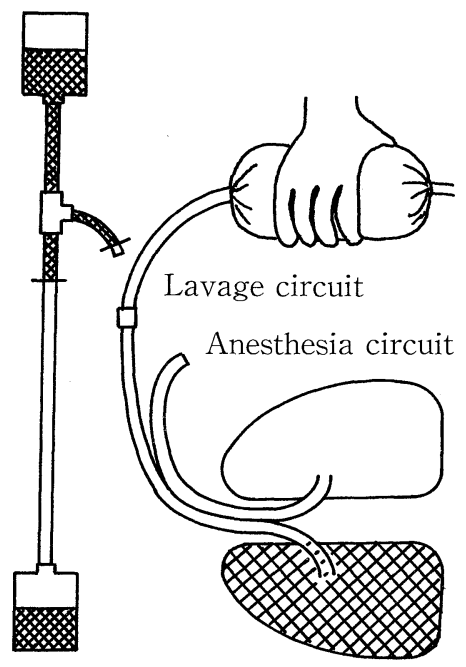

B

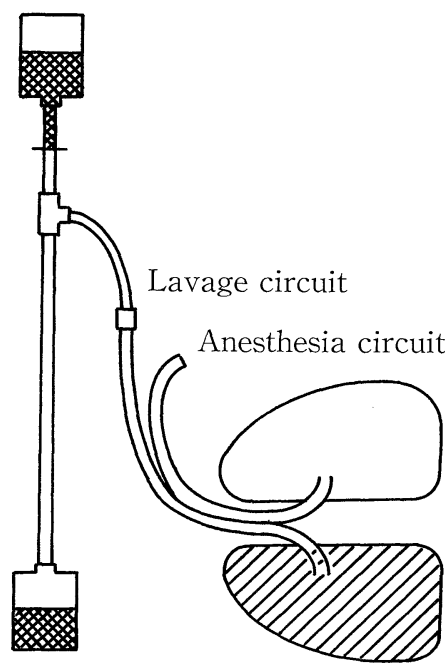

C

Figure 2. The method of the conventional lavage technique and the modified lavage technique introduced by Bingisser et al. (A) $1.0 \mathrm{l}$ of warm saline was instilled into the lavaged lung. (B) After passive recovery of half the amount of fluid, we manually ventilated the dependent lung 20 times with a tidal volume of $500 \mathrm{ml}$. (C) The fluid was then recovered passively. Conventional lavage technique. Repeat (A), (C) without (B) until the effluent becomes clear. Modified lavage technique introduced by Bingisser et al. Repeat (A), (B) and (C) until the effluent becomes clear.

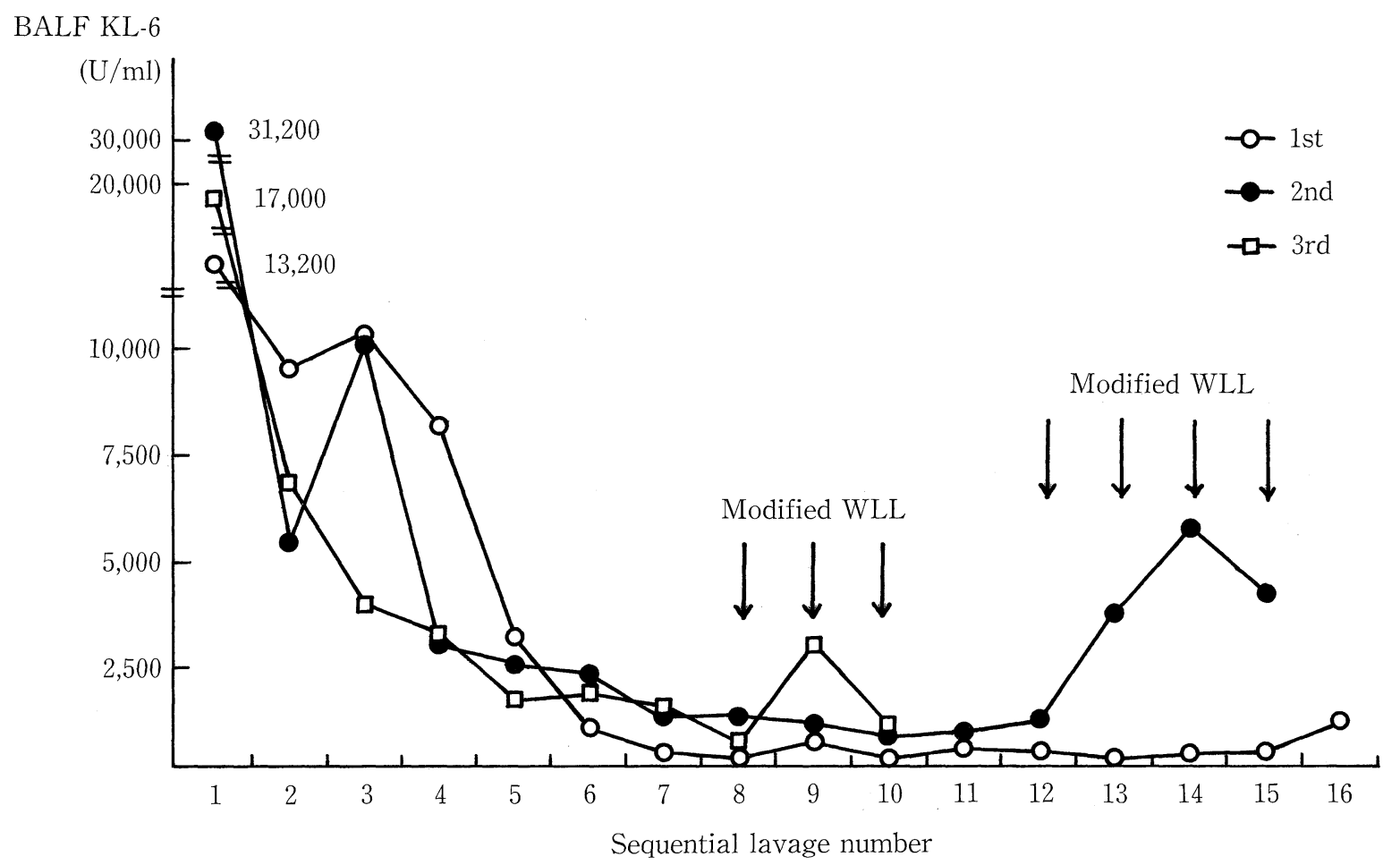

Figure 3. The levels of KL-6 in BALF during whole-lung lavages. KL-6 levels in BALF increased during lavages by the modified lavage technique of Bingisser et al, but not during those by the conventional lavage technique. WLL: wholelung lavage. 1st: conventional lavage technique only, 2nd: conventional lavage technique plus modified lavage technique of Bingisser, 3rd: conventional lavage technique plus modified lavage technique of Bingisser. 


\section{ISHIKAWA et al}

Serum KL-6

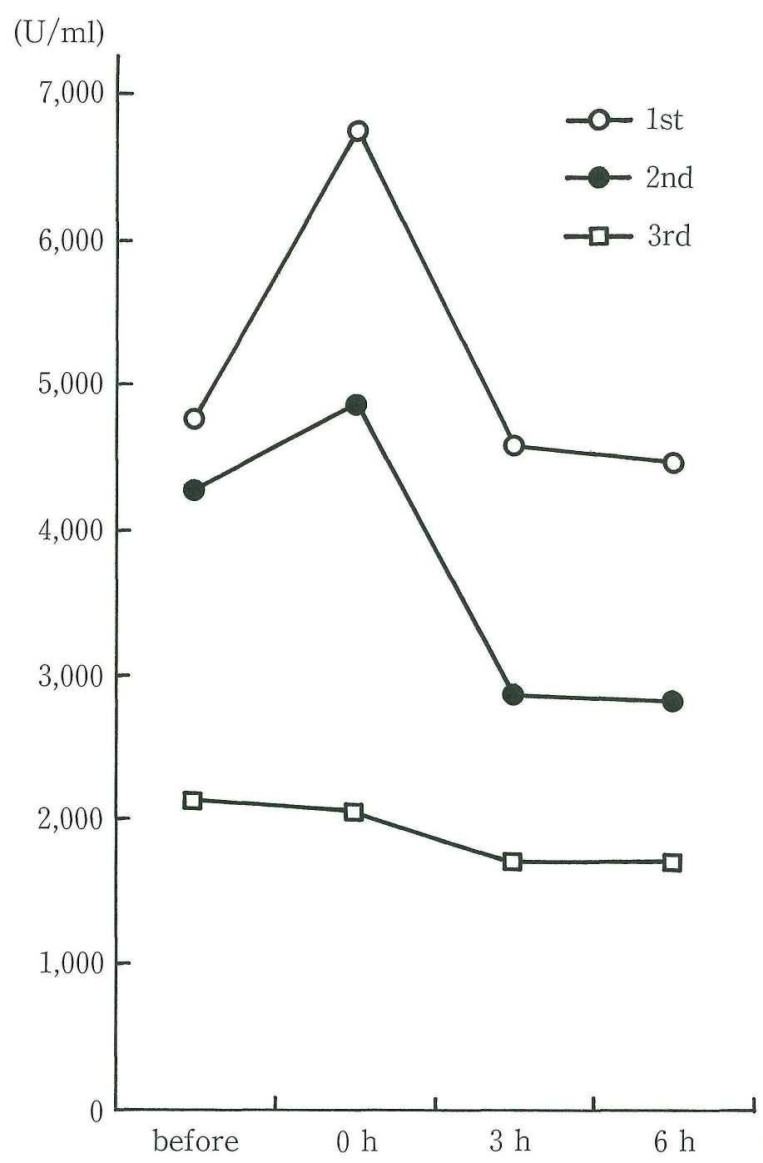

Figure 4. The levels of KL-6 in serum after whole-lung lavage, while the patient was ventilated mechanically. 1st: conventional lavage technique only, 2nd: conventional lavage technique plus modified lavage technique of Bingisser, 3rd: conventional lavage technique plus modified lavage technique of Bingisser. levels of KL-6 in BALF were extremely high before wholelung lavage, but decreased markedly with each whole-lung lavage. KL-6 levels in BALF were higher during lavages by the modified lavage technique of Bingisser et al, compared to the conventional technique (Fig. 3). Serum KL-6 levels increased transiently, but decreased within a few hours of completing the whole-lung lavage (Fig. 4). Disappearance of symptoms, improvement of the chest X-ray shadow, and recovery of pulmonary function were correlated with decreased levels of KL-6 in the serum.

Immunohistochemical staining with monoclonal KL-6 antibody (mouse $\mathrm{IgG}$ ) was performed on transbronchial biopsy specimens obtained at the time of diagnosis (Fig. 5). Pulmonary type II pneumocytes and proteinaceous material occupying the alveolar lumen were positive on KL-6 staining. There seemed to be no clear staining of alveolar macrophages or lymphocytes.

Twelve months after the final whole-lung lavage, he was asymptomatic with normal pulmonary function, normal arterial blood gas analysis, normal serum KL-6 levels, and resolution of the chest X-ray abnormalities. He has remained well to date.

\section{Discussion}

We showed the therapeutic effectiveness of a modified lavage technique of Bingisser et al in a patient with PAP. The efficacy of the lavage was objectively proven by monitoring KL-6 levels in his serum and BALF.

A recent report showed that manual ventilation applied during the second half of the drainage cycle is useful in PAP treatment; ventilation of a lung area partially filled with fluid allows better reopening of closed airspace and more efficient removal of proteinaceous materials (6). However, to our best knowledge, this was the only report of an individual with PAP

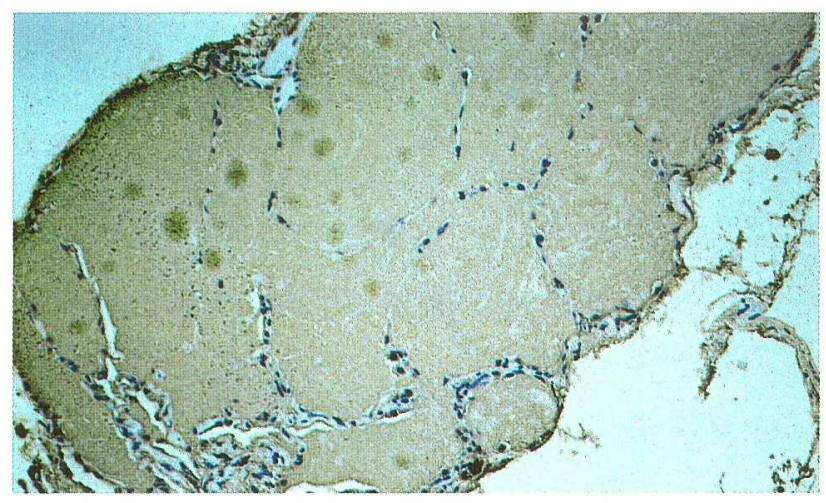

A

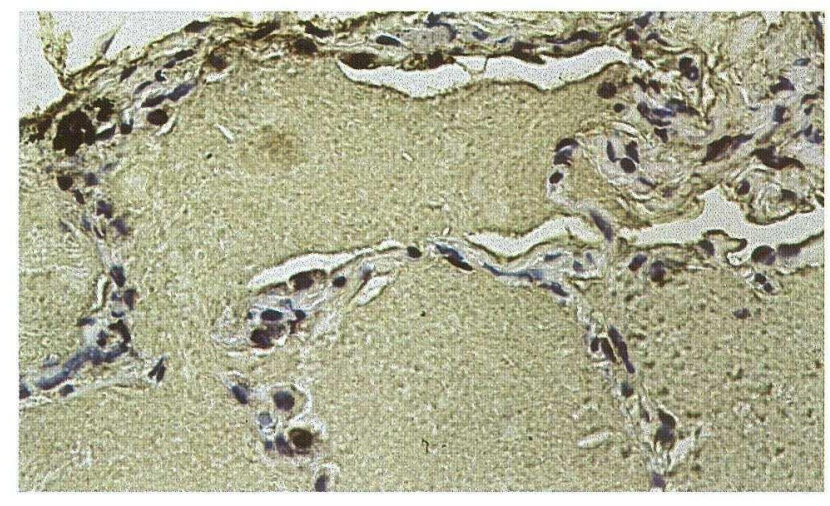

B

Figure 5. Immunohistochemical staining of the transbronchial biopsy specimen obtained at the time of diagnosis with monoclonal KL-6 antibody. (A) Proteinaceous material occupying the alveolar lumen was positive on KL-6 staining ( $\times 100$ ). (B) Pulmonary type II pneumocytes were positive on KL-6 staining $(\times 400)$. 
who had failed to improve by the conventional lavage technique. We therefore performed the same lavage technique on the presented case. During lavage by the modified lavage technique of Bingisser et al, the retrieved washing fluid became milky and KL-6 levels in BALF increased. These results may be explained by the fact that lavage by the modified lavage technique of Bingisser can remove proteinaceous materials from the distal airways and alveoli more effectively than lavage by the conventional technique. We concluded that we should be encouraged to try the modified lavage technique of Bingisser for the treatment of PAP patients who were not improved by conventional lavage technique.

In this case, serum KL-6 levels were markedly elevated. Levels of KL-6 in BALF were also elevated and were found to be higher than those in serum, consistent with previously reported data $(10,11)$. KL-6 is consistently present in the epithelial lining fluid (ELF), which is alveolar fluid from the distal airways and alveoli lavaged by BAL. In order for KL- 6 level in serum to increase, there must be a mechanism through which KL-6 in ELF is absorbed into the circulation. Since KL-6, which has a long and slender structure, is a large glycoprotein, a large passageway is needed for the absorption of KL-6 from ELF into the circulation, in contrast to small proteins like albumin. In patients with IP, KL-6 in ELF is easily absorbed into the circulation due to alveolar-capillary permeability as a result of damage to the alveolar epithelium (12-14). On the other hand, since epithelium undergo repeated reproduction and death even in healthy people, KL-6 in ELF is absorbed into the circulation through the passageway then produced. The present case, however, did not have fibrosis, but instead had hypertrophy of pulmonary type II pneumocytes. Since the immunohistochemical study of the present case showed that regenerating pulmonary type II pneumocytes and proteinaceous material expressed high levels of KL-6, the increased KL-6 level in ELF may be related to regenerating pulmonary type II pneumocytes. Furthermore, we speculate that pulmonary type II pneumocytes produce proteinaceous material, which then fills the alveoli in patients with PAP. Although the possibility that alveolar epithelial injury is caused by hypertrophy of pulmonary type II pneumocytes cannot be denied, we consider that the mechanism responsible for the increased serum KL-6 level in patients with PAP differs from that in patients with IP. We speculate that in PAP patients the markedly elevated level of KL-6 in the serum is due to the markedly elevated level of KL-6 in ELF and not to alveolar-capillary permeability.

We found that serum KL-6 levels were increased or maintained at high levels a few hours after completing the wholelung lavage, and then dropped rapidly. It was previously reported that patients with PAP who underwent lavage by the conventional lavage technique and were then ventilated mechanically, had increased surfactant protein A (SP-A) concentrations in BALF until the second hour after lavage and then the level decreased rapidly (15). There are several possible explanations for the change of serum KL-6 levels. 1) Since mechanical ventilation induces alveolar epithelial damage, the alveolar-capillary permeability to KL-6 might be increased. 2) Pressure from the mechanical ventilation might aid the transient flux of KL-6 from ELF into the circulation. However, since KL-6 in ELF is gradually cleared from the airways by therapeutic lavage, the flux of KL-6 from the ELF to the circulation eventually decreases. 3) The metabolism of KL-6 may be very rapid. At the moment, the mechanism responsible for the change in serum KL-6 levels remains unclear and these possibilities need to be explored further.

In summary, we reported the effectiveness of the modified lavage technique of Bingisser et al for a patient with PAP. Furthermore, determination of serum and BALF KL-6 levels was useful for assessing the treatment effect and disease activity.

\section{References}

1) Claypool WD, Rogers RM, Matuschak GM. Update on the clinical diagnosis, management, and pathogenesis of pulmonary alveolar proteinosis (phospholipidosis). Chest 85: 550-558, 1984.

2) Shah PL, Hansell D, Lawson PR, Reid KB, Morgan C. Pulmonary alveolar proteinosis: clinical aspects and current concepts on pathogenesis. Thorax 55: 67-77, 2000

3) Ramirez J, Schultz RB, Dutton RE. Pulmonary alveolar proteinosis: a new technique and rationale for treatment. Arch Intern Med 112: 419431, 1963.

4) Prakash UB, Barham SS, Carpenter HA, Dines DE, Marsh HM. Pulmonary alveolar phospholipoproteinosis: experience with 34 cases and a review. Mayo Clin Proc 62: 499-518, 1987.

5) Goldstein LS, Kavuru MS, Curtis-McCarthy P, Christie HA, Farver C, Stoller JK. Pulmonary alveolar proteinosis: clinical features and outcomes. Chest 114: 1357-1362, 1998.

6) Bingisser R, Kaplan V, Zollinger A, Russi EW. Whole-lung lavage in alveolar proteinosis by a modified lavage technique. Chest 113: 1718-1719, 1998.

7) Kohno N, Akiyama M, Kyoizumi S, Hakoda M, Kobuke K, Yamakido M. Detection of soluble tumor-associated antigens in sera and effusions using novel monoclonal antibodies, KL-3 and KL-6, against lung adenocarcinoma. Jpn J Clin Oncol 18: 203-216, 1988.

8) Kohno N, Inoue Y, Hamada H, et al. Difference in sero-diagnostic values among KL-6-associated mucins classified as cluster 9. Int J Cancer Suppl 8: 81-83, 1994.

9) Stahel RA, Gilks WR, Lehmann HP, Schenker T. Third International Workshop on Lung Tumor and Differentiation Antigens: overview of the results of the central data analysis. Int J Cancer Suppl 8: 6-26, 1994.

10) Nakajima M, Manabe T, Niki Y, Matsushima T. Serum KL-6 level as a monitoring marker in a patient with pulmonary alveolar proteinosis. Thorax 53: 809-811, 1998.

11) Takahashi T, Munakata M, Suzuki I, Kawakami Y. Serum and bronchoalveolar fluid KL-6 levels in patients with pulmonary alveolar proteinosis. Am J Respir Crit Care Med 158: 1294-1298, 1998.

12) Inoue $Y$, Barker E, Daniloff E, Kohno N, Hiwada K, Newman LS. Pulmonary epithelial cell injury and alveolar-capillary permeability in berylliosis. Am J Respir Crit Care Med 156: 109-115, 1997.

13) Kohno N, Awaya Y, Oyama T, et al. KL-6, a mucin-like glycoprotein, in bronchoalveolar lavage fluid from patients with interstitial lung disease. Am Rev Respir Dis 148: 637-642, 1993.

14) Kohno N. Serum marker KL-6/MUC1 for the diagnosis and management of interstitial pneumonitis. J Med Invest 46: 151-158, 1999.

15) Alberti A, Luisetti M, Braschi A, et al. Bronchoalveolar lavage fluid composition in alveolar proteinosis. Early changes after therapeutic lavage. Am J Respir Crit Care Med 154: 817-820, 1996. 